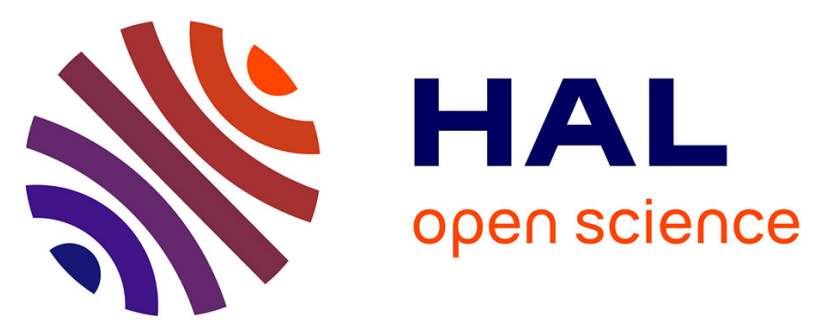

\title{
Suivi des donneurs apparentés : recommandations de la Société francophone de greffe de moelle et de thérapie cellulaire (SFGM-TC)
}

Alice Polomeni, Marjorie Culat-Farhat, Yohan Desbrosses, Christelle Andrianne, Malika Ainaoui, Evelyne Baillie, Nelly Bancillon, Natachade Bentzmann, Salaheddine Bouya, Elodie Duteil, et al.

\section{To cite this version:}

Alice Polomeni, Marjorie Culat-Farhat, Yohan Desbrosses, Christelle Andrianne, Malika Ainaoui, et al.. Suivi des donneurs apparentés: recommandations de la Société francophone de greffe de moelle et de thérapie cellulaire (SFGM-TC). Bulletin du Cancer, 2019, 107 (1), pp.S94-S103. 10.1016/j.bulcan.2019.02.006 . hal-02432790

\section{HAL Id: hal-02432790 \\ https://hal.sorbonne-universite.fr/hal-02432790}

Submitted on 31 Jan 2020

HAL is a multi-disciplinary open access archive for the deposit and dissemination of scientific research documents, whether they are published or not. The documents may come from teaching and research institutions in France or abroad, or from public or private research centers.
L'archive ouverte pluridisciplinaire HAL, est destinée au dépôt et à la diffusion de documents scientifiques de niveau recherche, publiés ou non, émanant des établissements d'enseignement et de recherche français ou étrangers, des laboratoires publics ou privés. 


\section{Suivi des donneurs apparentés : recommandations de la Société francophone de greffe de moelle et de thérapie cellulaire}

\section{(SFGM-TC)Related donors follow-up: Guidelines from the Francophone Society of Bone Marrow Transplantation and Cellular Therapy (SFGM-TC)}

Alice Polomeni, Marjorie Culat-Farhat, Yohan Desbrosses, Christelle Andrianne, Malika Ainaoui, Evelyne Baillie, Nelly Bancillon, Natachade Bentzmann, Salaheddine Bouya, Elodie Duteil, Christine Fraysse, Dominique Issarni, Agnès Ruscassie, Séverine Sauze, Jean-Baptiste Thibert, Ibrahim Yakoub-Agha, Catherine Faucher

\section{Résumé en Français :}

Le développement des greffes haplo-identiques amène à un recours de plus en plus important aux donneurs apparentés : de 2005 à 2015, ce recours s'est accru de 291\%. Audelà des coûts financiers moins importants, le don intrafamilial présente également l'avantage d'une disponibilité plus rapide du donneur, évitant ainsi des risques de rechute avant la procédure.

En dépit des recommandations existantes, on constate une diversité des pratiques dans les différents centres français quant aux modalités organisationnelles de prise en charge, aux critères de recrutement, qualification et suivi des donneurs apparentés.

La Société Française de Greffe de Moelle et Thérapie Cellulaire a fait des propositions concernant le consentement et les critères de non-qualification des donneurs apparentés de cellules souches hématopoïétiques. Un livret a été conçu par un groupe multidisciplinaire afin de faciliter l'information sur le don et sur l'allogreffe.

Dans le présent article, prenant en compte à la fois les impératifs médicaux de surveillance et les contraintes organisationnelles des centres greffeurs, nous proposons des recommandations pour le suivi des donneurs apparentés. Quelques outils (documents d'information, questionnaires) sont présentés.

Mots clés: donneur apparenté, suivi post-don, allogreffe de cellules souches hématopoïetiques, standardization prise en charge.

\section{Résumé en anglais}

The development of haplo-identical transplants brings to an increased resort to related donation: from 2005 till 2015, this resort increased by $291 \%$. Beyond the less important financial costs, the related donation also presents the advantage of a faster availability of the donor, avoiding risk of relapse before the procedure.

In spite of the existing recommendations, we notice a diversity of the practices among French centers as for the organizational modalities of care, for recruitment criteria, qualification and follow-up of related donors. 
French Society of Marrow Transplant and Cellular Therapy proposed recommendations concerning the consent and non-qualification criteria of related donors. A multidisciplinary group to facilitate the information about Hematopoietic stem cells donation and transplantation conceived a notebook.

In the present article, we propose recommendations for post-donation follow-up, taking into account at the same time medical imperatives and organizational constraints of transplantation teams. Some tools are also presented. 
Key words : related donor ; post donation follow-up ; hematopoietic stem cell transplantation ; care standardisation.

\section{Etat actuel de la question}

Le développement des greffes haplo-identiques amène à un recours de plus en plus important aux donneurs apparentés : de 2005 à 2015, ce recours s'est accru de 291\% [1]. $\mathrm{Au}$-delà des coûts financiers moins importants, le don intrafamilial présente également l'avantage d'une disponibilité plus rapide du donneur, évitant ainsi des risques de rechute avant la procédure.

Néanmoins, le don intrafamilial soulève des questions éthiques et cliniques spécifiques. Sur le plan éthique, les modalités organisationnelles du don - marquées notamment marquées par l'absence d'anonymat du don de CSH du fait du lien de parenté entre donneur et receveur - peuvent compromettre le statut volontaire et libre du don. [2].

Sur le plan clinique, les recommandations concernant le don de CSH, ne sont pas encore systématiquement appliquées pour les donneurs apparentés: « les critères d'éligibilité des donneurs apparentés sont moins stricts: la limite d'âge varie, diverses comorbidités sont acceptées, les doses de facteur de croissance, le nombre de prélèvements (y compris pour les DLI - Donor Lymphocytes Injection) et le volume recueilli ne sont pas préfixés » [3]. Les différences de prise en charge entre donneurs apparentés et non-apparentés illustrent le conflit d'intérêt opérant au sein des équipes médicales qui prennent en charge à la fois le patient et le donneur. Cette pratique courante au niveau international peut amener les équipes médicales à ne pas considérer de façon objective les risques médicaux et psychosociaux pour le donneur apparenté $[4,5]$.

Les risques médicaux sont dus aux procédures de cytaphérèse et/ou de prélèvement de moelle osseuse. Les risques psychosociaux sont liés d'une part aux conditions de libre choix vis-à-vis du don (pression médicale, familiale ou sociale) et, d'autre part, à l'obligation morale ressentie et à l'investissement affectif du donneur apparenté.

Sur le plan médical, il est admis que «le don de cellules souches hématopoïétiques (CSH) est associé à un nombre réduit, mais réel, d'événements indésirables » [6]. Des données rassurantes ont été recueillies quant aux risques d'hémopathies malignes suite à l'utilisation des facteurs de croissance [7], mais une surveillance est conseillée.

Notons que les données sur les incidents et événements indésirables se rapportent essentiellement aux donneurs non-apparentés, alors que les effets secondaires de la 
procédure sont plus fréquents chez les donneurs apparentés, probablement du fait de différences dans les critères et qualification et des variations procédurales [8].

Parmi ces critères variables, on peut souligner notamment la limite d'âge pour le don. Les données de l'étude RDsafe, étude prospective, multicentrique, indiquent cependant que l'âge n'est pas associé à une qualité de vie inférieure chez les donneurs de CSH entre 60 et 76 ans [9].

Alors que l'état de santé des donneurs non-apparentés est évalué par un questionnaire médical régulier et que les événements indésirables sont signalés, le suivi post-don des donneurs apparentés n'est pas systématiquement organisé [10].

La World Marrow Donor Association (WMDA) propose un suivi standardisé à court terme (à compléter pour les évènements survenus avant $\mathrm{J} 30$ post-don) à travers une numération formule sanguine (NFS) et un questionnaire de santé; ce même questionnaire sera utilisé pour le suivi à long terme (de cinq à dix ans après le don) [10]. Dans les centres affiliés à l'EBMT, ces données sont recueillies à travers la Donor Follow-up Form de la base Promise, ce qui permet d'en assurer la traçabilité.

Pour le suivi des donneurs, les recommandations de la SFGM-TC en 2010 étaient les suivantes (11):

? 0 Hémogramme à $\mathrm{J} 8,1$ et 6 mois.

0 Ferritinémie à 1 mois en cas de don de moelle.

? Information du médecin traitant sur type de don et suivi nécessaire.

? Consultation recommandée à $\mathrm{J} 30$, sinon contact téléphonique (en cas de problème = circuit de biovigilance).

? Questionnaires (à remplir par le donneur) le jour du prélèvement, à 1 mois, puis tous les ans.

Il est intéressant de noter que, malgré les données existantes sur l'impact psychologique du don $[12,13]$, cet aspect n'est pas systématiquement pris en compte dans la prise en charge des donneurs apparentés de CSH.

Une enquête réalisée auprès des infirmiers coordinateurs de greffe français montre que ces professionnels considèrent que le suivi post-don ne devrait pas se limiter à des aspects médicaux, mais inclure une évaluation systématique de l'état psychologique et de la qualité de vie des donneurs apparentés [14]. Cette évaluation pourrait s'avérer particulièrement pertinente dans le cadre des greffes haplo-identiques avec des donneurs ascendants ou descendants, susceptibles d'avoir un impact particulier sur les liens intergénérationnels. $[15,16]$.

Une étude française sur la prise en charge des donneurs fait état de leur satisfaction quant aux soins précédant et au cours du prélèvement, mais signale une frustration quant au suivi post-don [17]. Le sentiment d'être négligés est présent chez certains donneurs - ce qui est en accord avec d'autres études sur cette population [13]. 
Soulignons qu'au niveau des recommandations internationales, aucune orientation précise n'est posée concernant l'évaluation et le suivi psychologique des donneurs apparentés de $\mathrm{CSH}$, alors que dans le cadre du don d'organe solide (rein et foie), des conférences de consensus explicitent clairement les items pour la sélection des donneurs, proposant un guide d'entretien et des échelles validées $[18,19]$ pour l'évaluation des dimensions suivantes :

1. Motivation et processus de décision :

- pression/coercition au don

- relation donneur/receveur

- ambivalence

2. Ressources personnelles

- vulnérabilité

- attentes quant à l'impact du don sur les relations intrafamiliales

- mécanismes de défense et d'adaptation

3. Psychopathologie

- troubles cognitifs

- troubles de la personnalité

- troubles anxio-dépressifs (antécédents)

4. Soutien social

- situation socio-économique

- soutien familial et amical

5. Intégration des informations

- compréhension des effets secondaires, risques médicaux et impact psychologique du don

- compréhension du processus de la greffe (receveur) : attentes

- compréhension des aspects éthiques (libre choix, rétractation, engagement)

Les recommandations de la SFGM-TC à propos de la non-qualification des donneurs mentionnent qu'une anxiété importante (suscitée par la sélection pour le don et par la connaissance des effets secondaires du prélèvement), ainsi qu'un manque de fiabilité potentielle du donneur sont susceptibles de justifier une non-qualification. Il est conseillé que le risque de non-présentation le jour prévu pour le don soit évalué en colloque singulier, associé à une consultation psychologique si nécessaire [8].

Face à ces constats, la question du suivi des donneurs apparentés, tenant compte des aspects médicaux et psychosociaux, se pose. Ce suivi doit prendre en compte à la fois les réglementations et recommandations existantes [10,20,21] et la réalité des moyens disponibles dans les centres affiliés à la SFGM-TC, qui prennent en charge à moyens constants, un nombre croissant de donneurs apparentés.

\section{Questions posées}

Le suivi post-don doit cibler à la fois les risques médicaux et psychosociaux, dans une 
perspective de dépistage et de prévention. L'adhésion du donneur à cette démarche est indispensable et doit être favorisée par les informations données à la fois au donneur et à son médecin traitant sur la nécessité de ce suivi.

La pertinence de l'évaluation psychologique pré-don et les modalités du soutien psychologique ont été discutées, suscitant des questions sur l'approche de l'impact psychique du don par les équipes prenant en charge les donneurs apparentés.

A ce propos, le problème du conflit d'intérêts a été abordé lorsque les mêmes soignants sont amenés à prendre en charge donneur et receveur. L'intérêt et la faisabilité d'une prise en charge complètement indépendante (Etablissement Français du Sang ou Registre Français de Greffe de Moelle) ont été évoqués.

\section{Méthodologie :}

Cet atelier a été conduit selon la méthodologie des ateliers d'harmonisation des pratiques de la SFGM-TC [22]. Ce travail d'harmonisation est nécessaire du fait de la diversité et de l'évolution des connaissances et des pratiques. La réflexion sur l'adaptation des recommandations aux réalités des différents centres est indispensable, afin d'améliorer la qualité de la prise en charge des patients et, en l'occurrence, des donneurs apparentés. Une revue bibliographique a permis d'établir l'état de la question et de concevoir une enquête afin de connaître les modalités organisationnelles de prise en charge des donneurs apparentés dans les centres greffeurs de la SFGM-TC. Le questionnaire (annexe 1) a été construit sur la base de l'outil conçu par le Nurses Group de l'EBMT pour l'enquête européenne auprès des coordinateurs de greffe [23] et a été envoyé par mailing aux centres de la SFGM-TC.

Nous avons examiné les procédures actuelles, ainsi que les recommandations de l'atelier 2011, afin de proposer une démarche simplifiée, avec des outils pragmatiques et d'appropriation facile à la fois pour la clinique et pour l'organisation d'une base de données sur les donneurs apparentés de CSH.

\section{Résultats}

Vingt-six centres registrés à la SFGM-TC ont répondu, dont quatre pédiatriques. Les résultats sont présentés au tableau 1 et concernent 22 centres de greffes adultes. En effet, la problématique du don intrafamilial et ses procédures présente des spécificités dans le champ pédiatrique que nous ne traiterons pas dans cet article.

La majorité $(70 \%)$ des vingt-deux centres adultes ont été accrédités par le référentiel JACIE (Joint Accreditation Committee of International Society of Cell and Gene Therapy and European Bone Marrow Tranplantation Group). Dans 56\% des centres, le nombre 
de donneurs apparentés reçus par an est inférieur à 20, dans $36 \%$ des cas, il se situe entre 20 et 50; dans deux centres ce nombre s'élève à 80/an.

Questionnés sur l'existence d'un circuit indépendant pour donneurs et receveurs, 39\% des centres répondent négativement, 78\% affirment que les soignants (médecin, infirmier coordinateur de greffe, psychologue, assistante sociale) sont impliqués dans la prise en charge des donneurs et des receveurs, $86 \%$ d'entre eux participent à la réunion de concertation pluridisciplinaire au cours de laquelle sont discutées les indications de greffe. Notons néanmoins que dans $57 \%$ des cas, l'évaluation du donneur est réalisée par un médecin différent que celui qui prend en charge le receveur.

Le don intrafamilial est abordé avec le patient principalement par l'hématologue référent (48\%) ou par le médecin greffeur (41\%). Les donneurs potentiels sont contactés dans 31\% des cas par le patient. Ils sont informés sur le don et sur la greffe avant le typage HLA (Human Leukocyte Antigen)dans 68\% des centres, mais la plupart d'entre eux ne disposent pas d'un support écrit (livret) spécialement conçu pour cette information.

Les résultats du typage HLA sont communiqués au patient (46\%), à tous les donneurs typés (28\%) par l'hématologue, le médecin greffeur ou l'infirmière coordinatrice de greffe (IDEc). Un entretien d'information pour le donneur compatible est organisé majoritairement au moment du bilan pré-don (68\%). La table 2 décrit la liste des examens effectués lors du bilan pré-don.

Dans $82 \%$ des cas, des critères sont fixés par le centre pour l'éligibilité du donneur ; une limite d'âge est établie dans $57 \%$ des centres. Le pourcentage des donneurs non-validés au cours des deux dernières années ne dépasse pas $10 \%$ dans la majorité des centres $(58 \%)$ et est inférieur à $25 \%$ dans $37 \%$ des cas. Les données prises en compte pour l'évaluation médicale des donneurs apparentés dans les différents centres sont décrites au tableau 2. Lors du bilan pré-don, le donneur rencontré la plupart du temps un médecin et une IDEc, mais d'autres professionnels sont mentionnés (anesthésiste, IDE d'aphérèse, psychologue); un entretien psychologique est proposé dans $78 \%$ des centres lors du bilan pré-don. Un suivi post-don est organisé par $89 \%$ des centres. Les modalités sont diverses - sur le plan des moyens utilisés (contact téléphonique, questionnaire, consultation médicale) et des et des moments choisis (J0 à J7, J 30, J360); seuls trois centres effectuent un suivi jusqu'à cinq ans post-don (voir figure 1). Seuls $60 \%$ des entres ont répondu à la question sur les plaintes signalées par les donneurs lors du suivi post-don : majoritairement, il s'agit de douleurs et fatigue, mais des problèmes organisationnels et financiers, ainsi que le retentissement psychologique sont mentionnés. 
En résumé, nous notons un écart entre les recommandations et les pratiques concernant notamment l'existence de circuits indépendants donneurs/receveurs, la remise des résultats HLA au patient et les modalités de suivi post-don.

\section{Recommandations :}

Nous considérons que la prise en charge des donneurs apparentés devrait tendre, d'une façon générale, à s'approcher de celle des donneurs non-apparentés. La standardisation des outils de surveillance semble indispensable au recueil des données susceptibles d'être colligés sur les bases de données Promise/Macro. Le financement d'un temps de data manager pour la saisie de ces données serait susceptible d'être pris en charge par le FAG, venant en plus du temps dédié et déjà financé pour la saisie des données patients.

Soulignons que nos recommandations concernent les informations recueillies auprès des donneurs apparentés, certaines informations strictement médicales, notamment une déclaration obligatoire en cas d'événement indésirable grave, restent du domaine de la biovigilance.

Information aux donneurs et aux médecins traitants

L'information proposée au donneur apparenté doit attirer son attention sur l'intérêt du suivi post-don, sur les plans médical et psychologique. En effet, souvent l'impact psychologique du don est négligé, notamment dans le cadre du don intrafamilial où la problématique est souvent centrée sur le receveur.

La SFGM-TC a émis des recommandations sur l'information et le consentement du donneur, incluant la question du suivi et de la transmission des données recueillies [24, 25]. Ces informations sont aussi mentionnées dans le livret d'information pour le donneur, conçu collectivement dans un des ateliers d'harmonisation de l'année 2016 [26]. Une invitation explicite y est faite au donneur de faire appel aux psychologues tout au long du processus du don - y compris dans la période post-don.

Evaluation et suivi psychologique

La pertinence de l'utilisation des échelles de qualité de vie et/ou des échelles autoadministrées pour évaluer l'état psychique des donneurs au cours du suivi à court et long terme a fait débat.

D'une part, le recours à ces outils de mesure imposerait qu'une évaluation psychologique soit réalisée lors du bilan pré-don ou lors du prélèvement afin que les données recueillies soient interprétables. Or, cette évaluation pourrait susciter une anxiété supplémentaire chez le donneur, qui pourrait craindre une non-qualification du fait de sa condition psychique. 
D'autre part, même si l'argument de l'objectivation de l'évaluation et du suivi (à court et long terme) des aspects psychologiques par des outils standardisés peut être soutenu, l'insuffisance des moyens humains pour traiter ces informations rend actuellement inenvisageable ce type de procédure.

L'évaluation psychologique du donneur se fait de façon implicite au cours du processus du don, lors des entretiens avec l'IDEc et/ou le médecin responsable du prélèvement. Les équipes des centres greffeurs semblent sensibilisées à la dimension psychologique du don intrafamilial, puisqu'un entretien psychologique est souvent proposé (cf supra).

Par ailleurs, nous soulignons l'impact psychologique du don dans le courrier au médecin traitant (cf infra) qui, si nécessaire, pourrait adresser le donneur à des professionnels compétents, y compris la psychologue du centre greffeur si le donneur le souhaite et réside dans les environs.

Ainsi, nous ne proposons pas d'évaluation spécifique sur l'impact psychologique du don lors du suivi, hormis une question sur son état psychologique dans le questionnaire-socle. Procédure et outils du suivi post-don Nous recommandons un suivi post-don simplifié, basé sur le questionnaire utilisé par le Registre France de Greffe de Moelle et soutenu par le lien avec le médecin traitant. La procédure est conforme au référentiel JACIE.

Le suivi immédiat consiste en l'administration d'un questionnaire post-don (annexe 2) et la prescription d'une NFS à être réalisée au cours du premier mois suivant le prélèvement. Un courrier-type est adressé au médecin traitant (annexe 3) afin de l'informer sur la procédure de prélèvement, les possibles effets secondaires, ainsi que sur l'impact psychologique du don. En effet, il nous est apparu pertinent d'encourager le recours au médecin traitant comme interlocuteur privilégié pour les donneurs apparentés, tel que propose Culat-Frahat [17].

Ensuite, des questionnaires sont envoyés au donneur à J30, à un an et jusqu'à cinq ans postdon (annexes 4 et 5 ).

Le recours au médecin traitant, associé aux questionnaires réguliers envoyés aux donneurs, dans le respect des orientations JACIE, semble répondre à la fois à la nécessité d'une surveillance du donneur et aux contraintes institutionnelles en termes de moyens humains actuellement disponibles dans les centres greffeurs.

\section{Questions résiduelles}


Diverses questions ont été abordées, sans qu'un consensus puisse s'établir sur des recommandations précises puisqu'elles concernaient la prise en charge du donneur apparenté de façon plus large.

La première question a trait avec la possibilité de constituer une base de données recueillant les informations sur les donneurs apparentés. Les questionnaires envoyés au cours du suivi seront examinés par les centres greffeurs; néanmoins, ces données pourraient être saisies à travers le Donor Follow-up Form [27] dans la base internationale Promise, coordonnée par l'EBMT afin de constituer une source fiable pour des études rétrospectives, à l'instar de celles réalisées pour les donneurs non-apparentés. Le problème du financement d'un temps data-manager pour ce travail reste à examiner, et pourrait être proposé dans le cadre du forfait annuel greffe, à modifier pour intégrer le data management dédié aux donneurs. Le problème des modalités organisationnelles de prise en charge du donneur apparenté a été discuté. Les recommandations sur la nécessité d'un circuit indépendant de celui du receveur sont formelles [10, 20,21]. Néanmoins, des problèmes logistiques restent à résoudre, notamment dans le cas des centres ayant une petite activité de greffe. Notons également que même dans les centres avec un parcours indépendant, les professionnels qui prennent en charge le donneur sont impliqués indirectement dans les soins du receveur, ne serait-ce de par leur participation aux réunions de concertation pluridisciplinaire. Le débat à ce sujet fait état de deux types de proposition : d'une part, la prise en charge des donneurs apparentés par les registres de donneurs anonymes; d'autre part, la création d'unités spécifiques pour les donneurs apparentés, complètement séparés des unités de greffe [2830].

Un centre pédiatrique français met actuellement en place une prise en charge du donneur par le Service de Pédiatrie générale du même Centre Hospitalo-Universitaire. L'évaluation de cette expérience pourrait apporter des éléments pour une éventuelle réorganisation du parcours du donneur apparenté également dans les unités de greffe adulte.

La question de l'évaluation et du suivi psychologique du donneur apparenté reste également ouverte. D'une part, l'absence d'une base de données fiable et le manque d'études prospectives, multicentriques, ne permettent pas de statuer sur la nécessité d'une évaluation et suivi systématiques par un psychiatre et/ou un psychologue. D'autre part, les moyens humains pour effectuer ce travail sont actuellement insuffisants.

Les auteurs font part de leur préoccupation quant au recours aux donneurs haploidentiques ascendants ou descendants, soulignant à partir de leur expérience clinique, la spécificité de ce type de don intrafamilial qui tend à s'accroître de façon exponentielle. Des études sont nécessaires pour mieux évaluer l'impact psychologique des greffes haploidentiques sur le donneur et sur le receveur, afin de contribuer à la discussion sur le choix du donneur (lorsque plusieurs sont compatibles) et de leur proposer une prise 
en charge adaptée. Les présentes recommandations seront donc à actualiser selon les résultats de ces études.

\section{Déclaration de liens d'intérêts :}

Les auteurs ne déclarent pas de conflit d'intérêt

La SFGM-TC remercie les partenaires industriels pour leur soutien financier qui ont permis la réussite de cette neuvième édition des ateliers d'harmonisation des pratiques :

ABBVIE, BIOTEST, CELGENE, CHUGAI, GILEAD, JAZZ PHARMACEUTICALS, KEOCYT, MACOPHARMA, MALLINCKRODT THERAKOS, MSD FRANCE, NOVARTIS, SANOFI.

\section{Références:}

1. Passweg JR, Baldomero H, Badera P, Basak GW, Bonini C, et al for the European Society for Blood and Marrow Transplantation (EBMT)Is the use of unrelated donor transplantation leveling off in Europe? The 2016 European Society for Blood and Marrow Transplant activity survey report. Bone Marrow Transplantation 2018; 53:1139-1148.

2. Billen A, Madrigal JA, Shaw BR . A review of hematopoietic stem cell donation experience: is there room for improvement? Bonne Marrow Transplant 2014; 49:729-736.

3. Halter JP, VanWalraven SM, Worel N, Bengtsson M, Hägglund $\mathrm{H}$ et al. Allogeneic hematopoietic stem cell donation - standardized assessment of donor outcome data. Bonne Marrow Transplant 2013; 48: 220-225.

4. Anthias C, Shaw BE, Kiefer DM Liesveld JL, Yared J, et al. Significant Improvements in the Practice Patterns of Adult Related Donor Care in US Transplantation Centers. Biol Blood Marrow Transplant 2017; 22: 520-527.

5. Clare S, Mank A, Stone R, Davies M, Potting C, Apperley AF, on behalf of the Research Subcommittee of the EBMT Nurses Group. Management of related donor care: a European survey. Bonne Marrow Transplant 2010; 45; 97-101.

6. Halter J, Kodera Y, Ispizua AU, Greinix HT, Schmitz N, et al. Severe events in donors after allogeneic hematopoietic stem cell donation. Haematologica 2009; 94:94-101.

7. Shaw BE, Confer DL, Hwang W, Pulsipher MA. A review of the genetic and long-term effects of G-CSF injections in healthy donors: a reassuring lack of evidence for the development of haematological malignancies. Bone Marrow Transplant 2015;50(3):334-40. 
8. Boiron JM, Garban F, Audat F, Ader V, Andreu H et al. Critères de non-qualification des donneurs des cellules souches hématopoïétiques: recommandations de la Société francophone de greffe de moelle et de thérapie cellulaire (SFGM-TC). Bull Cancer 2017; 104S: S76-S83.

9. Switzer GE, Bruce J, Kiefer DM, Kobusingye H, Drexler R. et al. HRQoL among older related HSC donors ( $>60 \mathrm{yrs}$ ) is equivalent to that of younger related donors (18-60 yrs): an RDsafe study. Biol Blood Marrow Transplant 2017; 23 (1): 165-171.

10. Van Walraven S.M., Nicolo-de-Faveri G., Axdorph-Nygell U.A., Douglas K.W., Jones D.A. et al. on behalf of the WMDA Ethics and Clinical working groups Family donor care management: principles and recommendations. Bonne Marrow Transplant 2010; 45: 12691273.

11. Ateliers d'harmonisation SFGM-TC. Prise en charge des donneurs apparentés de CSH à court et long termes. 2010. http://www.sfgm-tc.com/recherche/harmonisation-despratiques-ateliers/29-pahpa-2010

12. Pillay B, Lee SJ, Katona L, De Bono S, Warren N, et al. The psychosocial impact of hematopoietic SCT on sibling donors. Bonne Marrow Transplant 2012; 47:1361-1365. 13. Garcia MC, Chapman JR, Shaw JP, Gottlieb DJ, Ralph A, et al. Motivations, Experiences, and perspectives of Bone Marrow and Peripheral Blood Stem Cell Donors: Thematic Synthesis of Qualitative Studies. Biol Blood Marrow Transplant 2013; 19:1046-1058. 14. Polomeni A, Bompoint C., Gomez A, Brissot E, Ruggeri AL, et al. Current practices for screening, consent and care of related donors in France: Hematopoietic Stem Cell Transplantation Coordinator Nurses' perceptions. Eur J Cancer Care 2017; 1-15. DOI:10.1111/ecc.12612

15. Van Walraven SM, Ball LM, Koopman HM, Switzer GE, Ropes-de Jong et al. Managing a dual role - experiences and coping strategies of parents donating haploidentical G-CSF mobilized peripheral blood stem cells to their children. Psycho-Oncology 2012; 21:168-175. 16. Polomeni A. « Enjeux éthiques et cliniques des thérapies innovantes: l'exemple de l'allogreffe de cellules souches hématopoḯtiques ». Revue Française d'Ethique Appliquée $2017 ;(4)$ : 91-103.

17. Cula-Farhat M. Evaluation du suivi des donneurs de cellules souches hématopoïétiques au sein du centre préleveur du CHRU de Besançon. Besançon : Université de Franche-Comté; 2018.

18. Ismail SY, Duerinckx N, van der Knoop MM, Timmerman L, Weimar W, Dobbels F, on behalf of the ELPAT Psychological Care for Living Donors and Recipients Working Group. Toward a Conceptualization of the Content of Psychosocial Screening in Living 
Organ Donors: An Ethical Legal Psychological Aspects of Transplantation Consensus. Transplantation 2015; 99: 2413-2421.

19. Potts S, Vitinius F, Erim Y, Gazdag G, Gribble R, et al. Mental health assessment of altruistic non-directed kidney donors: An EAPM consensus statement. Journal of Psychosomatic Research 107 (2018) 26-32

20. JACIE. International standards for hematopoietic cellular therapy product collection, processing and administration. https://www.ebmt.org/jacie-standards/7th-editioneffective-june-1st-2018

21. O'Donnell PV, Pedersen TL, Confer DL, Rizzo JD, Pulsipher MA, et al. Practice patterns for evaluation, consent, and care of related donors and recipients at hematopoietic cell transplantation centers in the United States. Blood 2010; 115(24):5097-101.

22. Tipton R, Yakoub-Agha I: [How we harmonize HSCT clinical practices among the SFGMTC centers]. Bull Cancer 2016; 103:S193-S197.

23. Polomeni A, Bompoint C, Badoglio M, Strnger J, Eeltink C. et al. Hematopoietic stem cell transplantation coordinator nurses' perceptionsof related donor care. Bone Marrow Transplantation Supplement 43rd Annual Meeting of the European Society for Blood and Marrow Transplantation 2017 ; 52 : S517-S528.

24. Thibert JB, Polomeni A, Yakoub-Agha I, Bordessoule D. Considérations générales et éthiques pour le recueil d'un consentement éclairé : recommandations de la Société Francophone de Greffe de Moelle et de Thérapie Cellulaire (SFGM-TC) Bull Cancer 2016;103: S207-S212.

25. Bruno B, Thibert JB, Bancillon N, Desbos A, Fawaz A, et al. Greffe de cellules souches hématopoïétiques - Information et consentement des donneurs et des receveurs: recommandations de la Société francophone de greffe de moelle et de thérapie cellulaire (SFGM-TC) Bull Cancer 2016; 103: S198-S200

26. Polomeni A, Tardieu L, Ainaoui M, Andrianne C, Bancillon N, et al. Un support d'information pour les donneurs apparentés de cellules souches hématopoïétiques : recommandations de la Société francophone de greffe de moelle et de thérapie cellulaire (SFGM-TC). Bull Cancer 2017; 104 : S71-S75

27.https://www.ebmt.org/sites/default/files/migration_legacy_files/document/DonorOutc omeForms.pdf

28. Anthias C, van Walraven SM, Sorensens, de Faveri GN, Fechter M, et al. Related hematopoietic cell donor care : is there a role for unrelated donor registries ? Bonne Marrow Transplant, 2015; $50: 637-641$. 
29. Muramatsu H, Kimura S, Ichinohe T, Ashihara E, Ishikawa T, et al. Consulting clinic for related family donors in hematopoietic stem cell transplantation. Bonne Marrow Transplant 2008 ; 41: 1073-1075.

30. Kisch A, Dykes J, Lindmark A, Lenhoff S. A proposed plan for the management of adult sibling donors. Bone Marrow Transplantation 2008; 42: 357-358. 